arXiv:0706.0514 [hep-th]

\title{
Runaway in the Landscape
}

\author{
Katrin Becker ${ }^{a}$, Melanie Becker ${ }^{a, b}$ and Johannes Walcher ${ }^{c}$ \\ a Texas A 89 University, College Station, TX 77843, USA \\ ${ }^{b}$ Harvard University, Cambridge, MA 02138, USA \\ c Institute for Advanced Study, Princeton, NJ 08540, USA
}

\begin{abstract}
We consider flux compactifications of type IIB string theory on the mirror of a rigid Calabi-Yau. In special cases, these models are dual to the type IIA flux vacua with runaway direction in flux space. We show that new weak coupling AdS solutions can be found for large complex structure, while Minkowski solutions with all moduli stabilized are confined to be at strong coupling. The existence of these solutions, as found in a previous work, is nevertheless guaranteed by a non-renormalization theorem of the type IIB flux superpotential. Based on our results, we are led to the conjecture that supersymmetric runaway directions in flux space are always accompanied by a spectrum of moduli masses reaching down to the AdS scale. This could be violated in a non-supersymmetric situation.
\end{abstract}

June 2007

Email: kbecker@physics.tamu.edu, mbecker@physics.tamu.edu, walcher@ias.edu 


\section{Introduction}

One of the most important lessons we learned about flux compactifications in the past couple of years is that moduli fields of the internal geometry can be stabilized in these compactifications (for a review and an extensive list of references see [1], [2]). In a vast majority of the compactifications the addition of non-perturbative effects coming from gluino condensation or wrapped branes is needed in order to stabilize all the moduli. This is not totally unexpected [3] if one insists on working with a small parameter controlling a semi-classical expansion.

It therefore came as a surprise when an explicit model with non-zero masses for all geometric moduli fields at the classical level (i.e. in terms of fluxes only) appeared in [4] in the context of the massive type IIA theory. One of the most interesting aspects of this proposal is that it exhibits a direction in the space of flux configurations on the internal manifold which is not constrained by tadpole cancellation while maintaining $\mathcal{N}=1$ supersymmetry. Along this direction, one gains back a small parameter, the inverse flux number, which can be used for a perturbative expansion.

One may wonder if by going to the large flux limit one isn't throwing out the baby with the bathwater. Namely, it has been emphasized that the masses of the geometric moduli appearing in [4] are actually proportional to the cosmological constant of the four-dimensional anti de Sitter (AdS) space. In other words, their Compton wavelengths are comparable to the AdS radius. So just as "tachyons" with negative mass squared above the Breitenlohner-Freedman bound, those moduli can be considered as effectively massless for all practical purposes. Note that this does not stand in the way of the fact that the vacua of [4] are indeed four-dimensional because the Kaluza-Klein scale is parametrically larger that the inverse AdS radius.

The question arises whether this sort of behavior of moduli masses will bear out in any runaway direction in the landscape in which one is gaining parametric control over the expansion by dialing some fluxes to be large. In this paper, we will find evidence that the answer to this question is in the affirmative. We will address this question by extending the type IIB models recently proposed in [5. Similar in spirit as in [4] the models proposed in [5] have all moduli stabilized in terms of fluxes only, but in distinction to those of 4], were constructed without any small parameter at all. Control is provided by a powerful non-renormalization theorem for the flux superpotential.

Our first aim is to deform the models of [5] and show that they exhibit a runaway 
direction in flux space similar to [4]. In the context of this model it will then be shown in section 4 that parametric control over the expansion is indeed closely related to masses of the size of the AdS scale.

The advantage of working in type IIB is that one has technical control over a larger variety of fluxes than in type IIA. This greater amount of freedom in dialing the fluxes might lead to the expectation that the masses of moduli are not necessarily linked to the AdS scale. We will not be able to realize this expectation, and although we will come relatively close, we view our failure as evidence that it might simply not be possible to do so. In fact, we conjecture that for any sequence of supersymmetric weakly coupled string vacua reaching out to the boundary of moduli space the masses of some of the moduli fields are of the order of the cosmological constant 1 Moduli fields emerging in compactifications of string theory to AdS or Minkowski space can be stabilized at best at strong coupling and could only be described explicitly if a weakly coupled dual conformal field theory with a large mass gap can be constructed.

Our conjecture is somewhat reminiscent of one of the general swampland conjectures of [7] according to which boundary regions of moduli space are always signaled by the appearance of new light fields in the spectrum. This might help to further delineate features of the swampland [6], and also gives support to the conjecture that the number of string vacua passing the most basic of physical cuts is finite [7], [9].

It is expected that the situation changes after supersymmetry is broken (and the vacua are uplifted to de Sitter (dS) space), because then moduli fields may acquire masses that in principle could be much larger than the cosmological constant. Flux compactifications offer several mechanisms for supersymmetry breaking. In the type IIB context, the main mechanism that uplifts the AdS vacua to dS space is the one proposed in [10] by using anti-branes. This is close to the local models of [11]. In [13], 14] it was proposed that a system of branes and anti-branes on the resolved conifold is holographically dual to a flux configuration with appropriate sign flips on the deformed conifold. The advantage of the dual formulation is that supersymmetry can be broken spontaneously to $\mathcal{N}=0$ in terms of only fluxes. One may wonder if supersymmetry can be broken spontaneously for the compact models considered herein. In

\footnotetext{
${ }^{1}$ It seems likely that such a generic constraint on AdS vacua could be effectively studied using general properties of the putative dual conformal field theories. Such an approach was advocated by many people and was used concretely in [8] in constraining the mass gap for $\mathrm{AdS}_{3}$ vacua using the modularity of 2d CFT partition function.
} 
the type IIA context, such a mechanism was considered in [16]. (See [15] for a general discussion of uplifting in IIA.) The type IIB version, on which we shall elaborate in Section 5, has two ingredients (which are mirror dual to those considered in [16]). To begin with, one turns on only fluxes of RR type, which by themselves break supersymmetry spontaneously, but lead to a runaway potential for the dilaton. This runaway can then be stabilized similarly to [10] in the presence of suitable non-perturbative effects, and lead to a vacuum with a positive cosmological constant! We will also show that masses of moduli can become much larger than the scale of the cosmological constant in this type of vacua.

A byproduct of our analysis is that the models first constructed in [4] in type IIA in fact admit a mirror dual description in terms of a more familiar construction in type IIB. Namely, all the fluxes considered in [4] (in their explicit examples, not necessarily the general case) in fact transform under mirror symmetry into ordinary RR and NSNS three-form fluxes. The mirror transform can be done using the SYZ approach by taking into account that the $3 \mathrm{~T}$-dualities are done along the directions in which there is no $H_{N S}$ flux. This claim might be surprising if one recalls that in the usual type IIB construction, supersymmetric flux configurations have an imaginary self-dual (ISD) property and therefore lead to a positive definite contribution to the D3-brane tadpole. As a consequence, there should not be any supersymmetric runaway direction in flux space which is not constrained by the tadpole. The reason for the unexpected existence of a runaway direction in flux space is an unusual prefactor in the Kähler potential for the dilaton. This prefactor is peculiar to the non-geometric models we consider here, and is predicted by mirror symmetry as we explain in some detail. Supersymmetric fluxes in the non-geometric type IIB models are not constrained to be ISD.

This paper is organized as follows. In section 2 we review some of the relevant formulas of the type IIB model constructed in [5] that will be needed in the later sections. Due to its importance in connection to the existence of strong coupling solutions, we will elaborate the non-renormalization theorem presented in [5] in some more detail. In section 3 we present the equations that constrain weak coupling type IIB flux vacua. In section 4 it is shown that AdS type weak coupling solutions can be found with masses of the order of the cosmological constant. Minkowski space solutions are shown to live at strong coupling. In section 5 we present a mechanism to break supersymmetry in terms of fluxes and argue that the masses of states can in principle be much larger than the cosmological constant once the theory is uplifted to dS space. 
In section 6 we present our conclusions and open questions.

\section{A Reminder of the Basics}

In this section we summarize some material concerning the type IIB non-geometric model constructed in [5] that will be relevant in the later sections.

The concrete example we are interested in is a non-geometric model described in terms of an orientifold a of Landau-Ginzburg (LG) model. In [5] it was shown that several types of four-dimensional supersymmetric vacua (Minkowski as well as AdS) exist at the Fermat point of this model. Even though these vacua emerge at strong coupling their existence is warranted due to a powerful non-renormalization theorem for the type IIB superpotential [22], 23], 24], [5]. We will discuss the non-renormalization theorem in some detail in this section. In order to compute e.g. the explicit value of the masses of moduli, it is of importance to find weak coupling solutions, as will be done in the following sections.

\subsection{The model}

We would like to compactify the type IIB theory on the mirror of a rigid Calabi-Yau manifold 2with $h_{11}=0$ and $h_{21}=84$. The theory we are interested in is constructed in terms of a LG model based on nine minimal models and worldsheet superpotential

$$
W=\sum_{i=1}^{9} x_{i}^{3},
$$

divided by a $\mathbb{Z}_{3}$ symmetry generated by

$$
g: x_{i} \rightarrow \omega x_{i},
$$

with $i=1, \ldots, 9$ and $\omega=e^{\frac{2 \pi i}{3}}$. Having $h_{11}=0$ the model has no Kähler moduli (or hypermultiplets except for the universal hypermultiplet) so that it is intrinsically non-geometric.

Constraints following from the tadpole cancellation condition can be satisfied by considering an orientifold of this LG model which can be obtained by dividing by

\footnotetext{
${ }^{2}$ We restrict here to the model with $h_{21}=84$ for concreteness, even though a model with $h_{21}=90$ was also constructed in [5]. Our subsequent analysis will be similar for the second model constructed in $[5]$.
} 
worldsheet parity $\Omega_{B}$ dressed with a holomorphic involution $P$ in space-time

$$
P:\left(x_{1}, x_{2}, x_{3} \ldots x_{9}\right)=-\left(x_{2}, x_{1}, x_{3}, x_{4}, \ldots, x_{9}\right)
$$

Complex structure deformations emerge as deformations of $W$ and a basis of such deformations is given in terms of the invariant monomials of the chiral ring

$$
x_{i} x_{j} x_{k}
$$

where $i \neq j \neq k \neq i$. There are $h_{21}=63$ monomials which are invariant under the $\mathbb{Z}_{3}$ action as well as the orientifold action. There are no Kähler structure deformations so that the size of the manifold is not a modulus. Due to this fact, the supergravity approximation is (strictly speaking) not valid and CFT techniques need to be used to describe the internal theory. Nevertheless the vacua that we will present are perturbatively and non-perturbatively stable. As argued in [5] this is due to the existence of a non-renormalization theorem of the type IIB flux superpotential, which will be presented in the next subsection in some detail.

The above LG model can also be thought of as a toroidal orbifold, where the orbifold group is a non-geometrically acting quantum symmetry [25] [5]. The type IIA mirror of this model is however geometric. It can be represented in terms of a torus $T^{6}=\left(T^{2}\right)^{3}$ divided by a $\mathbb{Z}_{3} \times \mathbb{Z}_{3}$ symmetry generated by

$$
\begin{aligned}
& g_{12}:\left(z_{1}, z_{2}, z_{3}\right) \rightarrow\left(\omega z_{1}, \omega^{-1} z_{2}, z_{3}\right), \\
& g_{23}:\left(z_{1}, z_{2}, z_{3}\right) \rightarrow\left(z_{1}, \omega z_{2}, \omega^{-1} z_{3}\right),
\end{aligned}
$$

where $z_{i}, i=1,2,3$ are the coordinates of the torus.

From this representation it becomes obvious that the mirror of our model is related to the toroidal model presented in [4], though the action of the orbifold symmetry is somewhat different. Similarly as in [4] our model has three bulk moduli that correspond to the complex structures of the three tori, while the remaining moduli correspond to blow up modes.

Here we will focus on stabilizing the bulk moduli and we shall assume that blow up modes have been stabilized at a different scale. This will already give us some useful information about the moduli space. In the next section it is shown how the equations constraining the bulk moduli of the toroidal orbifold of [4] emerge as a special case of our equations. 


\subsection{Non-renormalization theorem}

There are several arguments supporting the non-renormalization theorem of the superpotential for complex structure moduli in the type IIB theory. First indirect evidence came from the matrix model calculation of [23]. Here we shall elaborate a more direct argument based on supersymmetry that was already presented in [5]. In general, the superpotential of an $\mathcal{N}=1$ theory receives non-perturbative corrections. However, the particular $\mathcal{N}=1$ theory we are interested in has its origin in a theory with $\mathcal{N}=2$ supersymmetry, so that corrections to the superpotential are absent.

Compactifying the type IIB theory on a Calabi-Yau threefold will in general result in a theory with $\mathcal{N}=2$ supersymmetry in $d=4$ containing $h^{21}$ vector multiplets and $h^{11}+1$ hypermultiplets (where the ' 1 ' denotes the universal hypermultiplet). The $\mathcal{N}=2$ supersymmetry can be broken to $\mathcal{N}=1$ by adding a Fayet-Iliopoulos term [26], [27], [28]. We are interested in the case $h^{11}=0$ which describes a theory with $h^{21}$ abelian vector multiplets and the universal hypermultiplet. To illustrate the argument it suffices to consider the simpler case of a theory with only one abelian $\mathcal{N}=2$ vector multiplet, which has an expansion in terms of $\mathcal{N}=1$ superfields

$$
\Phi=\phi^{(1)}(\tilde{y}, \theta)+\sqrt{2} \tilde{\theta}^{\alpha} \phi_{\alpha}^{(2)}(\tilde{y}, \theta)+\tilde{\theta}^{\alpha} \tilde{\theta}_{\alpha} \phi^{(3)}(\tilde{y}, \theta),
$$

where $\tilde{y}^{\mu}=x^{\mu}+i \theta \sigma^{\mu} \bar{\theta}+i \tilde{\theta} \sigma^{\mu} \overline{\tilde{\theta}}$, and $\theta, \tilde{\theta}$ and their complex conjugates are superspace coordinates. The action for the $\mathcal{N}=2$ vector then takes the form

$$
\int d^{2} \theta d^{2} \tilde{\theta} \mathcal{F}_{0}(\Phi)
$$

where $\mathcal{F}_{0}$ is the $\mathcal{N}=2$ prepotential. Supersymmetry can be broken by adding an FI term

$$
\int d^{2} \theta d^{2} \tilde{\theta} \mathcal{F}_{0}(\Phi)+\xi D
$$

where $D$ is one of the three auxiliary fields of the $\mathcal{N}=2$ vector multiplet. Integrating out the auxiliary field exactly reproduces the $\mathcal{N}=1$ flux superpotential containing $H_{R R}$

$$
W=\int H_{R R} \wedge \Omega
$$

as $H_{R R}$ appears as an auxiliary field in the $\mathcal{N}=2$ vector multiplet. The superpotential is not renormalized neither perturbatively nor non-perturbatively as the $\mathcal{N}=2$ prepotential for vector multiplets is not renormalized [22]. One simple argument for this is that in $\mathcal{N}=2$ supersymmetric theories neutral hypermultiplets and vector multiplets 
do not couple and the dilaton is part of a hypermultiplet. Furthermore, $\alpha^{\prime}$ corrections can be excluded as they contain the size of the Calabi-Yau which is a Kähler modulus. Using the $S L(2, \mathbb{Z})$ symmetry of the type IIB theory we can write down the unique $S L(2, \mathbb{Z})$ invariant combination of the superpotential

$$
W=\int G \wedge \Omega
$$

where $G=H_{R R}-\tau H_{N S}$. The complete superpotential is then not renormalized as the $\mathcal{N}=2$ prepotential is not corrected. This argument is already enough to ensure the existence of Minkowski vacua, which are solutions to the equation $\partial_{i} W=0$, where ' $i$ ' denotes the moduli. Some more thought is needed to guarantee the existence of supersymmetric AdS vacua which are solutions to the equation $D_{i} W=\partial_{i} W+\partial_{i} K W=$ 0 . This is due to Kähler invariance [5]. Namely suppose we can choose the coordinates for the moduli $t_{i}$ in such a way that the Kähler potential has an expansion

$$
K=t_{i} \bar{t}_{i}+a_{i j} t_{i} \bar{t}_{j} f(t, \bar{t})
$$

where $t_{i}=\bar{t}_{i}=0$ describes our solution. Quantum corrections to $\partial_{i} K$ evaluated at $t_{i}=\bar{t}_{i}=0$ will affect the solution only by terms which are purely holomorphic

$$
\delta K=\delta f(t)+c c
$$

Such a correction can, however, be absorbed into the superpotential which is a holomorphic section of a line bundle $W \rightarrow \exp (-\delta f(t)) W$, so that the solution is unaffected.

The non-perturbative non-renormalization theorem of the type IIB superpotential is valid for models containing no Kähler moduli. The mirror type IIA interpretation of this result is that for models with only one three-cycle there are no non-perturbative corrections, as the only available three-cycle contains $H_{N S}$ flux. We will return to possible non-perturbative corrections in Section 5.

\section{IIB Flux Vacua at Weak Coupling}

In this section we derive the equations constraining the three bulk moduli. We shall see that mirror symmetry and the duality to the IIA model of [4] predicts a subtle correction to the dilaton Kähler potential in type IIB, which cannot be obtained by a Kaluza-Klein reduction, in accord with the fact that our model is not geometric in the first place. We will see that this correction opens new directions in the landscape 
leading to an infinite set of new AdS type vacua in the large complex structure limit! Minkowski vacua with stabilized moduli are unaffected and constrained to live at strong coupling [5].

\subsection{Deformations}

The first step in our construction is to go away from the Fermat point (2.1). More precisely, we shall be interested in the LG model with superpotential

$$
W=\sum_{i=1}^{9} x_{i}^{3}+a_{1} x_{1} x_{2} x_{3}+a_{2} x_{4} x_{5} x_{6}+a_{3} x_{7} x_{8} x_{9}+\cdots
$$

We intend to make $a_{1}, a_{2}, a_{3}$ large, and the dots are all other possible cubic monomials which however we will assume to be small compared with the $a_{i}$. The point of the deformation (3.1) is that in the limit of large $a_{i}$, the LG-model is precisely mirror to the rigid $T^{6}$ orbifold (2.5). This is clear from the standard LG description of the moduli space of $T^{2}$. The remaining deformations of $W$ correspond to blowup moduli from the $T^{6}$ perspective. By going to this region of moduli space, we are able to focus on the stabilization of the three "bulk moduli" $a_{1}, a_{2}, a_{3}$. (All other $T^{6}$ moduli are projected out by the orbifold.) Thus we reduce our computation to one in the neighborhood of three copies of the large complex structure limit of $T^{2}$. The twisted sector moduli can then in principle be stabilized by turning on fluxes through the blowup cycles. This can be justified in principle by an exact computation of the periods, which was done for the model at hand in [29], or alternatively along the lines of [4].

\subsection{Potentials for bulk moduli}

Let us denote the three (complex) bulk moduli as $t_{1}, t_{2}, t_{3}$. These moduli fields (which are related to the $a_{i}$ in (3.1) in the usual way [30], 31] ) represent the three complex structures of $T^{6}=\left(T^{2}\right)^{3}$. At this point we will write the formulas with all $t_{i}$ 's in place and consider the case $t_{1}=t_{2}=t_{3}$ later on for calculational simplicity (though we would like to conjecture that our conclusions also hold for the more generic situation).

Let us first describe the superpotentials and Kähler potentials that are needed to describe the constraining equations for the bulk moduli. The type IIB flux superpo- 
tential 3

$$
W=\int\left(H_{R R}-\tau H_{N S}\right) \wedge \Omega=W_{R R}-\tau W_{N S}
$$

is described in terms of complex structure moduli and the dilaton, while Kähler structure moduli do not appear. Here $\Omega$ denotes the holomorphic three form of the internal space and $\tau=C_{0}+i e^{-\phi}$ is the axio-dilaton combination. Let us rewrite this superpotential in terms of the bulk moduli by expanding the three form fluxes in terms of a cohomology basis dual to the symplectic basis $\left(A^{I}, B_{J}\right)$ of $H_{3}(M, \mathbb{Z})$. We choose the intersection numbers to satisfy

$$
A^{I} \cap B_{J}=-B_{J} \cap A^{I}=\delta_{J}^{I} \quad \text { and } \quad A^{I} \cap A^{J}=B_{I} \cap B_{J}=0 .
$$

The dual cohomology basis is denoted by $\left(\alpha_{I}, \beta^{J}\right)$ and satisfies

$$
\int_{A^{J}} \alpha_{I}=\int \alpha_{I} \wedge \beta^{J}=\delta_{I}^{J} \quad \text { and } \quad \int_{B_{J}} \beta^{I}=\int \beta^{I} \wedge \alpha_{J}=-\delta_{J}^{I} .
$$

In terms of this basis the fluxes can be expanded as

$$
\begin{aligned}
& H_{R R}=M_{0} \alpha_{0}+M_{2}\left(\alpha_{1}+\alpha_{2}+\alpha_{3}\right)-M_{4}\left(\beta^{1}+\beta^{2}+\beta^{3}\right)-M_{6} \beta^{0}, \\
& H_{N S}=N_{0} \alpha_{0}+N_{2}\left(\alpha_{1}+\alpha_{2}+\alpha_{3}\right)-N_{4}\left(\beta^{1}+\beta^{2}+\beta^{3}\right)-N_{6} \beta^{0},
\end{aligned}
$$

where the $M_{p}$ 's and $N_{p}$ 's are the flux numbers. Furthermore we take into account that the A-periods of $\Omega$ determine the coordinates on moduli space, while the B-periods determine the derivatives of the prepotential

$$
z^{I}=\int_{A^{I}} \Omega, \quad \mathcal{G}_{I}(z)=\int_{B_{I}} \Omega
$$

In the large complex structure limit the prepotential of the model takes the form [30]

$$
\mathcal{G}(z)=-\frac{1}{3 !} \kappa_{I J K} \frac{z^{I} z^{J} z^{K}}{z^{0}}
$$

where $\kappa_{I J K}$ are the Yukawa couplings. Since the model can be related to a torus $T^{6}$ the only non-vanishing Yukawa coupling is $\kappa_{123}=1$ (and symmetric permutations of the indices). The resulting superpotential is

$$
\begin{aligned}
& W_{R R}=-t_{1} t_{2} t_{3} M_{0}+\left(t_{1} t_{2}+t_{1} t_{3}+t_{2} t_{3}\right) M_{2}+\left(t_{1}+t_{2}+t_{3}\right) M_{4}+M_{6}, \\
& W_{N S}=-t_{1} t_{2} t_{3} N_{0}+\left(t_{1} t_{2}+t_{1} t_{3}+t_{2} t_{3}\right) N_{2}+\left(t_{1}+t_{2}+t_{3}\right) N_{4}+N_{6},
\end{aligned}
$$

\footnotetext{
3 Note that the model is not geometric, so that the integrals that follow have to be interpreted from the conformal fields theory point of view, as was done in [5].
} 
which is written in terms of the affine coordinates $t^{\alpha}=z^{\alpha} / z^{0}$.

Having the form of the superpotential we need the form of the Kähler potential for the axio-dilaton and bulk moduli. This potential takes the form

$$
K=-\log \left[i\left(t_{1}-\bar{t}_{1}\right)\left(t_{2}-\bar{t}_{2}\right)\left(t_{3}-\bar{t}_{3}\right)(\tau-\bar{\tau})^{4}\right] .
$$

We need to pause to explain the structure of the Kähler potential for the axio-dilaton, $-4 \log (\tau-\bar{\tau})$ as opposed to the familiar $-\log (\tau-\bar{\tau})$ (compare e.g. with the appendix of [32]). Recall that one way to define the model is to start on a large volume $T^{6}$, go to a symmetric point in Kähler moduli space and then orbifold in such a way as to project out all Kähler moduli (see Section 2.1). Let us rephrase this in supergravity language, as this allows for an easier comparison with type IIA analysis of [4]. Assume that we have a four dimensional $\mathcal{N}=2$ supergravity with some number $n_{h}$ of hypermultiplets, one of which is the universal hypermultiplet and some number $n_{v}$ of vector multiplets. In many cases, the moduli space of hypermultiplets contains a region in which the prepotential is cubic, and which can be derived by dimensional reduction from ten dimensional supergravity. Let us call it the cubic region. In type IIB this is simply the large volume regime. In type IIA, we have to be in the large volume and also in the "large complex structure limit".

We now break $\mathcal{N}=2$ to $\mathcal{N}=1$ by an orientifold action and fluxes. After the orientifold projection we remain with $n_{v}^{-} \mathcal{N}=1$ vector multiplets and $n_{h}+n_{v}^{+} \mathcal{N}=1$ chiral multiplets. As is customary, we will continue to call the $\mathcal{N}=1$ chiral multiplets which come from $\mathcal{N}=2$ vector multiplets as vector multiplet moduli and those which come from $\mathcal{N}=2$ hypermultiplets as hypermultiplet moduli.

Now it was shown in [33], [34] that after orientifold projection the Kähler potential for the hypermultiplets in the cubic region is of the form

$$
K=-\log (\tau-\bar{\tau})-2 \log e^{-3 \phi / 2} \kappa_{a b c} v^{a} v^{b} v^{c},
$$

where $v^{a}$ are some real coordinates on the hypermultiplet moduli space (Kähler moduli in type IIB) and $\kappa_{a b c}$ are the Yukawa couplings. See in particular chapter 3, section 3 of [34]. The main point of the discussion is that one should view the $v^{a}$ as the worldsheet couplings, whereas the holomorphic coordinates on the hypermultiplet moduli space from the spacetime point of view contain as real part the $\operatorname{Re}\left(T_{a}\right) \sim e^{-\phi / 2} v^{a}$, where $e^{-\phi}=\operatorname{Im}(\tau)$ is the dilaton. Therefore, in the large volume limit, the Kähler potential for the dilaton indeed reduces to just $-\log (\tau-\bar{\tau})$, as expected from a Kaluza-Klein reduction. 
Now on the torus the cubic expression (3.10) is exact (as there are no worldsheet instanton corrections). We can then go to the $\mathbb{Z}_{3} \times \mathbb{Z}_{3}$ symmetric point $v^{a}=1 / 2$, and orbifold. Since orbifolding is a worldsheet operation it projects the worldsheet variables $v^{a}$ to their value at the orbifold point but does not touch the dilaton directly. It then follows easily from (3.10) that after orientifold and orbifold the Kähler potential for the dilaton is

$$
K(\tau)=-4 \log (\tau-\bar{\tau}) .
$$

In a certain sense, one should view the factor of 4 as a small volume correction to the usual expression and which emerges for the type IIB non-geometric models. This correction can only be derived by mirror symmetry and not by analytic continuation from a geometric Kaluza-Klein reduction in type IIB! In fact, [33, 34] give a more general expression for the Kähler potential valid (in the supergravity approximation) throughout the hypermultiplet moduli space and this is what is used in [4] in their general analysis.

Notice that this non-geometric modification of the Kähler potential only affects AdS type vacua, because the determining equations for Minkowski vacua do not depend on the Kähler potential.

\subsection{Tadpole cancellation and supersymmetry constraints}

The single most interesting aspect of the modified Kähler potential for the axio-dilaton is that unbroken supersymmetry no longer requires the three-form flux to be imaginary self-dual (ISD) even in the absence of non-perturbative corrections. This renders the flux contribution to the tadpole non-positive definite and makes the tadpole cancellation condition less constraining than in the usual cases. This is essentially what allows the existence of the sequence of flux vacua with arbitrarily large flux numbers found in [4].

More concretely, using the explicit form of the flux superpotential the supersymmetry constraint for the axio-dilaton reads 4

$$
D_{\tau} W=-\frac{1}{\tau-\bar{\tau}} \int(3 G+\bar{G}) \wedge \Omega=0
$$

\footnotetext{
${ }^{4}$ As noted in the foot of page 10, the integrals here and below obtain their meaning from the CFT/LG description of our model.
} 
which is solved by

$$
\tau=\frac{1}{2} \frac{W_{R R}}{W_{N S}}\left(3-e^{i \varphi}\right) \quad \text { where } \quad \varphi=\operatorname{Arg}\left(\frac{W_{N S}}{W_{R R}}\right) .
$$

The supersymmetry constraint for the complex structure moduli is expressed in terms of a basis $\chi_{i}$ of harmonic $(2,1)$ forms

$$
D_{i} W=\int G \wedge \chi_{i}=0
$$

From this we see that unbroken supersymmetry requires the Hodge decomposition of $G$ to be

$$
G=A^{i} \chi_{i}+A^{0}(-3 \Omega+\bar{\Omega})
$$

where the $A$ 's are constants. As a result $G$ can have a component in the $(3,0)$ direction which is IASD, as opposed to the $(2,1)$ and $(0,3)$ components which are ISD. This violates the standard lore according to which supersymmetric three-form fluxes in Calabi-Yau compactifications of type IIB string theory are constrained to be $(2,1)$ and in particular ISD. Moreover, this renders the flux contribution to the tadpole non-positive definite. To see this we write the tadpole in the form

$$
\int H_{R R} \wedge H_{N S}=i e^{\phi} \int G \wedge \bar{G}
$$

Using that the metric on moduli space $g_{i \bar{j}}$ and the Kähler potential $K$ are represented by

$$
g_{i \bar{j}}=i e^{K} \int \chi_{i} \wedge \bar{\chi}_{j}>0 \quad \text { and } \quad i \int \bar{\Omega} \wedge \Omega=e^{K}>0,
$$

and are both positive definite we obtain a negative contribution to the tadpole from a particular component of $G$ namely

$$
i \int(-3 \Omega+\bar{\Omega}) \wedge(-3 \bar{\Omega}+\Omega)=-8 e^{K}<0 .
$$

Thus by turning on enough of this flux component we get an indefinite space of supersymmetric fluxes. This results in flux vacua with a finite tadpole in new directions of flux space. Some of these directions allow flux numbers tending to infinity. In the following we would like to discuss the properties of the string theory landscape along these directions. 


\section{Supersymmetric Solutions}

Our goal in this section is to search for supersymmetric solutions at weak coupling and for large values of the complex structure. We shall look for solutions with diagonal complex structure $\left(t_{1}=t_{2}=t_{3}\right)$ and will later conjecture that this simpler situation reproduces all the features of the more generic situation. For three equal complex structures the flux superpotentials take the form

$$
\begin{aligned}
& W_{R R}=-t^{3} M_{0}+3 t^{2} M_{2}+3 t M_{4}+M_{6}, \\
& W_{N S}=-t^{3} N_{0}+3 t^{2} N_{2}+3 t N_{4}+N_{6} .
\end{aligned}
$$

The Kähler potential for the bulk moduli and axio-dilaton is

$$
K=-\log \left[i(t-\bar{t})^{3}(\tau-\bar{\tau})^{4}\right] .
$$

Using this form of the potentials we shall now look for Minkowski as well as AdS type supersymmetric solutions.

\subsection{Minkowski space solutions}

In the following we will see that supersymmetric Minkowski space solutions do not emerge for large complex structure but are confined to finite value of the complex structure and strong coupling. These are the solutions presented in [5]. Groundstates corresponding to a four-dimensional Minkowski space are obtained as solutions of

$$
W=D_{\tau} W=D_{t} W=0,
$$

which can equivalently be written as

$$
W_{R R}=W_{N S}=0 \quad \text { and } \quad \tau=\frac{W_{R R}^{\prime}(t)}{W_{N S}^{\prime}(t)} .
$$

We are interested in finding physical solutions for which the imaginary parts of $t$ and $\tau$ are non-vanishing, because otherwise the solutions lie at the boundary of the moduli space. Since vanishing of the superpotentials (4.1) results in cubic equations with real coefficients, complex solutions of these equations only exist if the cubic polynomials $W_{R R}$ and $W_{N S}$ have two common complex conjugate roots, i.e. if they factorize according to

$$
\begin{aligned}
& W_{R R}=C(t-\alpha)(t-a)(t-\bar{a}), \\
& W_{N S}=D(t-\beta)(t-a)(t-\bar{a}),
\end{aligned}
$$


where $C, D, \alpha, \beta$ are real and $a$ is complex. These numbers are constrained by flux quantization condition. The complex structure is then determined from the zeros of a quadratic equation, or equivalently

$$
t=a=a_{1}+i a_{2}
$$

Moreover, taking into account Eqs. (3.4), (3.5), (4.3), (4.4) the tadpole can be written in the form

$$
\int H_{R R} \wedge H_{N S}=4\left(M_{2} N_{0}-N_{2} M_{0}\right)\left(a_{2}\right)^{2}
$$

Note that the coefficient in front of $\left(a_{2}\right)^{2}$ is integer and cannot be made arbitrarily small nor equal to zero (the lhs of the equation does never vanish in Minkowski). As a result $a_{2}$ is bounded by the $\mathrm{O} 3$ plane charge. In the concrete examples considered in [5] the largest value of the $\mathrm{RR}$ charge arising from an $\mathrm{O} 3$ plane was 12 . As a result Minkowski space solutions only exist if the imaginary part of the complex structure is small like for example in the solutions found in [5]. In the type IIA mirror these are solutions at small volume of the internal geometry, for which no perturbative control is expected. In the following we will see that AdS solutions do exist in the large complex structure limit.

\subsection{Constraints for AdS type solutions}

Supersymmetric flux configurations which allow a negative cosmological constant in the external space-time are the solutions of

$$
D_{\tau} W=D_{t} W=0
$$

Since the vanishing of $W$ is no longer required, solutions of AdS type are less constrained. It is useful to first rewrite the superpotential in a more practical manner. For this take into account that the equation $D_{\tau} W=0$ allows us to write $\tau$ in the form 5

$$
\tau=\frac{1}{2} \frac{W_{R R}}{W_{N S}}\left(3-e^{i \varphi}\right) .
$$

Inserting this form of $\tau$ into the superpotential we get a representation of the superpotential in terms of $W_{R R}$ up to a phase

$$
W=\frac{1}{2} W_{R R}\left(-1+e^{i \varphi}\right) .
$$

\footnotetext{
${ }^{5}$ It is sensible to divide by $W_{R R}$ or $W_{N S}$ as Minkowski solutions are not a subclass of the AdS type solutions derived in the following.
} 
Using Eqs. (4.9) and (4.10) as well as the form of the Kähler potential (4.2) it is easy to see that the constraint $D_{t} W=0$ takes the form

$$
\frac{\partial_{t} W_{R R}}{W_{R R}}-\frac{1}{2}\left(3-e^{i \varphi}\right) \frac{\partial_{t} W_{N S}}{W_{N S}}=-\frac{3}{2} \frac{1}{t-\bar{t}}\left(1-e^{i \varphi}\right) .
$$

This will be our starting point in the search for supersymmetric AdS configurations.

\subsection{Weak coupling AdS type solutions}

Using the ansatz (4.1) for the superpotentials and the constraints (4.9) and (4.11) we obtain the solution to the supersymmetry constraints. Different types of solutions are possible. Our aim is to present some concrete examples, leaving the search for the most general solution for future work.

\subsubsection{Constant $W_{N S}$}

The simplest solution of Eq. (4.11) is given by flux configurations in which $W_{N S}$ is constant, i.e.,

$$
W_{N S}=N_{6} \quad \text { while } \quad W_{R R}=-t^{3} M_{0}+3 t^{2} M_{2}+3 t M_{4}+M_{6},
$$

where the flux numbers $N_{0}, N_{2}$ and $N_{4}$ are set to zero. The corresponding fluxes given by Eq. (3.5) induce a contribution to the tadpole of the form

$$
\int H_{R R} \wedge H_{N S}=-M_{0} N_{6}
$$

which only constrains the flux numbers $M_{0}$ and $N_{6}$.

The modulus $t=t_{1}+i t_{2}$ is determined from Eq. (4.11) which for real and constant $W_{N S}$ takes the form

$$
\partial_{t} W_{R R}+\frac{3}{2} \frac{\operatorname{Im}\left(W_{R R}\right)}{\operatorname{Im}(t)}=0 .
$$

The imaginary part of this equation determines the real part of the complex structure

$$
t_{1}=\frac{M_{2}}{M_{0}}
$$

while the real part of the equation determines $t_{2}$

$$
t_{2}=\sqrt{\frac{5}{3}} \sqrt{-\frac{M_{2}^{2}}{M_{0}^{2}}-\frac{M_{4}}{M_{0}}} .
$$


The imaginary part of the axio-dilaton which follows from Eq. (4.9) is

$$
\tau_{2}=-\frac{24}{5} \frac{M_{0}}{N_{6}}\left(t_{2}\right)^{3} .
$$

Since $M_{2}, M_{4}$ and $M_{6}$ are not bounded by the orientifold charge they can be made arbitrarily large, giving us small parameters controlling the expansion, the inverse of the flux numbers. As a result solutions exist in the large complex structure and weak coupling limit, as becomes evident from the above expressions for $t_{2}$ and $\tau_{2}$. Further, the axion $\tau_{1}$ is fixed by Eq. (4.9). This example describes the solution discussed in [4] as can be seen by comparing to expressions (4.6), (4.20)-(4.22) of that papen. We thus see that the non-geometric type IIB model is mirror to the massive type IIA model of [4] for a particular choice of our flux quantum numbers! However in type IIB we have more freedom to dial the fluxes, so that more general solutions can be constructed. Let us see an example of this next.

\subsubsection{Non-constant $W_{N S}$}

Besides the solutions described in the previous paragraph there are more directions in the landscape parametrized by different combinations of flux numbers $N_{i}$ and $M_{i}$. One such example can be constructed in terms of flux configurations with

$$
W_{R R}=3 M_{2} t^{2}+M_{6} \quad \text { and } \quad W_{N S}=3 N_{4} t .
$$

The contribution of the fluxes to the tadpole is

$$
\int H_{R R} \wedge H_{N S}=3 M_{2} N_{4},
$$

and as a result the $\mathrm{O} 3$ plane charge limits the values of $M_{2}$ and $N_{4}$ while $M_{6}$ can be taken to be arbitrarily large. The imaginary part of the complex structure and the axio-dilaton are given by

$$
t_{2}=\sqrt{-\frac{M_{6}}{9 M_{2}}} \quad \text { and } \quad \tau_{2}=-8 i \frac{M_{2}}{N_{4}} \sqrt{t_{2}},
$$

while the corresponding axionic partners (described in terms of the real part of $t$ ) vanish. (But, as will be clear from the expressions for the mass matrix in the next

\footnotetext{
${ }^{6}$ The reader should not get confused by the counting of moduli, which is 36 in 4 and 63 for us. The precise statement is that a subclass of our models is mirror to [4]. Both models have 3 bulk moduli describing tori coordinates and only differ in the number of blow up modes, which are not considered here in detail.
} 
subsection, both $\tau_{1}$ and $t_{1}$ have a non-zero mass in those solutions.) From the result (4.20) we observe that by taking $M_{2}, N_{4} \sim O(1)$ and $M_{6}$ large we obtain a weak coupling solution in the large complex structure limit. The value of the moduli scale in a different manner with the fluxes as for the solutions in the previous subsection.

\subsection{The mass matrix}

In the following we would like to show that in the large complex structure limit a generic property of the mass matrix of the moduli fields at the supersymmetric AdS groundstates is that it is of the order of the space-time cosmological constant.

The masses of moduli fields are determined from the second derivatives of the scalar potential at the groundstate which are given by

$$
\begin{aligned}
& \partial_{\bar{b}} \partial_{a} V=e^{K}\left(D_{a} D_{c} W \overline{D_{d} D_{a} W} g^{c \bar{d}}-2 g_{a \bar{b}}|W|^{2}\right), \\
& \partial_{a} \partial_{b} V=-e^{K}\left(D_{a} D_{b} W\right) \bar{W} .
\end{aligned}
$$

Here the indices $a, b, \ldots$ label all the fields, i.e. the complex structure and the axiodilaton. To obtain these expressions we have repeatedly used $D_{a} W=0$ at the groundstate. For the diagonal ansatz (4.1), we find the following expression for the second Kähler derivatives of the superpotential at the supersymmetric groundstate

$$
\begin{aligned}
D_{\tau} D_{\tau} W & =-\frac{12 W}{(\tau-\bar{\tau})^{2}}, \\
D_{t} D_{\tau} W & =-D_{t} W_{N S}, \\
D_{t} D_{t} W & =-\frac{2(\tau-\bar{\tau})}{t-\bar{t}} \overline{D_{t} W_{N S}} .
\end{aligned}
$$

By introducing the parameters

$$
x=(\tau-\bar{\tau})(t-\bar{t}) \frac{D_{t} W_{N S}}{W} \quad \text { and } \quad y=(\tau-\bar{\tau})(t-\bar{t}) \frac{\overline{D_{t} W_{N S}}}{W}
$$

the canonically normalized mass matrix for the moduli $t, \tau$ written as a hermitian matrix whose entries are given in Planck units is

$$
M_{\text {phys }}^{2} / \Lambda_{A d S}=\left(\begin{array}{cccc}
\frac{2}{3}-\frac{10}{108}|x|^{2} & \frac{2}{9} y & -\frac{\bar{x}}{2 \sqrt{3}}-\frac{\bar{y} x}{9 \sqrt{3}} & \frac{\bar{x}}{6 \sqrt{3}} \\
\frac{2}{9} y & \frac{2}{3}-\frac{10}{108}|x|^{2} & \frac{x}{6 \sqrt{3}} & -\frac{x}{2 \sqrt{3}}-\frac{y \bar{x}}{9 \sqrt{3}} \\
-\frac{x}{2 \sqrt{3}}-\frac{y \bar{x}}{9 \sqrt{3}} & \frac{\bar{x}}{6 \sqrt{3}} & -\frac{7}{3}-\frac{1}{36}|x|^{2} & 1 \\
\frac{x}{6 \sqrt{3}} & -\frac{\bar{x}}{2 \sqrt{3}}-\frac{x \bar{y}}{9 \sqrt{3}} & 1 & -\frac{7}{3}-\frac{1}{36}|x|^{2}
\end{array}\right) .
$$


From this explicit expression we see that the essential parameter that controls the masses is $x$ from (4.23) (note that $y=\bar{x}$ up to a phase). So we need to look for solutions of $D_{t} W=0$ with large $|x|$ and large $t_{2}$.

Next we use that $W_{R R}$ and $W_{N S}$ have one real and a pair of complex conjugate roots. Since $W_{N S}$ is a polynomial of third degree in $t$, having small $x$ is only possible if $t$ is close to a zero of $W_{N S}$. Since moreover $t$ is a physical field, its imaginary part should be non-vanishing. By Eq. (4.11) we also need to be close to the zeros of $W_{R R}$. We write the factorization of the cubic polynomials (4.1) as

$$
\begin{array}{ll}
W_{R R}=C(t-\alpha)(t-a)(t-\bar{a}) & \alpha \text { real } \\
W_{N S}=D(t-\beta)(t-b)(t-\bar{b}) & \beta \text { real. }
\end{array}
$$

Using this ansatz Eq. (4.11) reduces to

$$
\frac{1}{t-\alpha}+\frac{1}{t-a}+\frac{1}{t-\bar{a}} \approx \frac{1}{2}\left(3-e^{i \varphi}\right)\left(\frac{1}{t-\beta}+\frac{1}{t-b}+\frac{1}{t-\bar{b}}\right)
$$

where we have neglected the term on the right hand side of Eq. (4.11) since it is subleading in the large complex structure limit. In order to satisfy our constraints, the parameters $a$ and $b$ need to have a large imaginary part, and we need to look for a solution for which the real and imaginary parts of $t-a, t-b$ are of order 1 . It is not difficult to see that Eq. (4.26) then reduces to

$$
(t-b) \approx(t-a) A(t)
$$

where we have introduced the variable $A(t)$ related to the phase $\varphi$ by

$$
A(t)=\frac{1}{2}\left(3-e^{i \varphi}\right) .
$$

Note that $\varphi$ is approximately given by

$$
e^{i \varphi} \approx e^{i \tilde{\varphi}}\left(\frac{t-b}{\bar{t}-\bar{b}}\right)\left(\frac{\bar{t}-\bar{a}}{t-a}\right) \quad \text { where } \quad e^{i \tilde{\varphi}}=\left(\frac{t-\alpha}{\bar{t}-\alpha}\right)\left(\frac{\bar{t}-\beta}{t-\beta}\right) .
$$

The solvability of Eq. (4.27) now depends on the value of the phase $\tilde{\varphi}$. It is not difficult to see that if $e^{i \tilde{\varphi}}$ is real Eq. (4.27) has trivial solutions only. On the other hand note that

$$
e^{i \tilde{\varphi}}=\left(\frac{t_{1}-\alpha+i t_{2}}{t_{1}-\alpha-i t_{2}}\right)\left(\frac{t_{1}-\beta-i t_{2}}{t_{1}-\beta+i t_{2}}\right) .
$$


Since in the fundamental domain of $S L(2, \mathbb{Z}),\left|t_{1}\right| \leq 1 / 2$, a complex phase can only be obtained in the large complex structure limit if $\alpha$ and/or $\beta$ are proportional to $t_{2}$. However, under this assumption the tadpole reduces to

$$
\int H_{R R} \wedge H_{N S}=M_{0} N_{6}-\frac{1}{3} M_{0} N_{0}+\frac{1}{3} M_{2} N_{4}-M_{4} N_{2} \approx C D \frac{2}{3}(\alpha-\beta) \operatorname{Im}(a)^{2}+O(\operatorname{Im}(a))
$$

which implies that $\alpha=\beta$ to leading order. This forces the phase factor $e^{i \tilde{\varphi}}$ to be real.

We conclude that it is not possible to keep the masses large in such a large flux limit in which the complex structure moduli become very large while maintaining supersymmetry and the tadpole cancellation condition. Notice that although we have not strictly imposed that the dilaton also runs to infinity, this would not be an independent constraint in our ansatz, in which $\tau \sim C / D$, see eq. (4.9). We could have reached weak coupling if the tadpole had been satisfied.

\section{Non-supersymmetric Solutions}

The supersymmetric AdS solutions of the previous section had moduli fields with masses that were too small (of the order of the AdS scale) so that these fields could be considered effectively as massless. We here want to explore the possibility that this situation is remedied after breaking supersymmetry by appropriate effects.

In a superficially similar situation, KKLT [10] proposed to uplift type IIB AdS vacua to dS space by adding anti-D3 branes. The resulting masses are of the order of the AdS scale before the uplift so by appropriate fine-tuning will be large compared to the positive cosmological constant after the uplift. Adding anti-D3-branes is certainly one possibility to uplift our AdS type vacua of Section 4 and to get masses of the right scale. However, anti-D3-branes break supersymmetry by hand and we would like to explore if a mechanism that breaks supersymmetry spontaneously (only with fluxes) can be found. Such a mechanism was proposed in [13, 14 for non-compact models. It was shown there that a model with branes and anti-branes is holographically dual to a model containing only fluxes after a geometric transition. There is a simple generalization of spontaneous supersymmetry breaking by fluxes for compact models, that makes use of corrections to the scalar potential.

It was pointed out in [16] within the context of type IIA compactifications on Calabi-Yau orientifolds that perturbative corrections to the Kähler potential generate a contribution to the potential for the dilaton which is similar to the contribution of anti- 
D3 branes to the scalar potential for the radial modulus in KKLT. Supersymmetry can be broken spontaneously instead of by anti-branes. It was further argued in [16] that membrane instantons [20] generate a non-perturbative correction to the superpotential of the dilaton, which resembles the non-perturbative correction to the superpotential for the radial modulus coming from gaugino condensation and wrapped D3 branes in KKLT.

Mirror symmetry implies that higher order terms in the type IIB theory result in perturbative corrections to the Kähler potential for the dilaton]. Membrane instantons correspond to $\mathrm{D}(-1)$ instantons in the type IIB theory. The AdS type models discussed in Section 4 contain no hypermultiplets (except for the universal one), and do not have non-perturbative corrections to the superpotential according to the nonrenormalization theorem. From the type IIA side this follows from the absence of membrane instantons, as the only available three-cycle contains $H_{N S}$ flux [21]. Of course, more complicated models having more than one three-cycle could receive nonperturbative corrections, which will be discussed next or there could be corrections arising from branes wrapping cycles or the whole Calabi-Yau which are forbidden in supersymmetric solutions but which could appear once supersymmetry is broken. It is quiet possible that perturbative corrections to the Kähler potential lift the AdS type vacua of Section 4 to $\mathrm{dS}$ space with moduli field masses of the right magnitude. Once the numerical coefficients of all relevant type IIB interactions are known, this can be checked explicitly.

It is curious that there is a second type of rather simple type IIB vacua that break supersymmetry spontaneously. As we shall see these vacua require no sinks of RR charge in order to satisfy the tadpole cancellation condition on a compact CalabiYau three-fold since they have $H_{N S}=08$. Consider the scalar potential of $\mathcal{N}=1$ supergravity in four dimensions

$$
V=e^{K}\left(g^{a \bar{b}} D_{a} W \overline{D_{b} W}-3|W|^{2}\right)
$$

where in our case $a, b$ label the axio-dilaton and complex structure moduli. It turns out that in this model moduli stabilization can be achieved using $\mathrm{RR}$ three-form fluxes

\footnotetext{
${ }^{7}$ One of these terms was used in [17] to correct the Kähler potential, but it is possible to have more contributions from the higher order terms in the action appearing in [18, [19].

${ }^{8}$ These supersymmetry breaking configurations will be further discussed in $[12$.
} 
only, i.e. by assuming the superpotential is of the form

$$
W=W_{R R}=\int H_{R R} \wedge \Omega .
$$

Since in this case $W$ is independent of $\tau$ it is a matter of simple manipulations to show that

$$
V=e^{K}\left(g^{i \bar{j}} D_{i} W_{R R} \overline{D_{j} W_{R R}}+\left|W_{R R}\right|^{2}\right),
$$

where $i, j$ label the complex structure moduli only. This expression which closely resembles the scalar potential for non-supersymmetric black holes is positive definite 9 so we can hope to get vacua with a positive cosmological constant. However, $\tau_{2}$ is not stabilized since the only dependence in $\tau$ appears in the overall factor $e^{K} \sim \operatorname{Im}(\tau)^{-4}$. This last factor causes the dilaton to run to weak coupling.

One way to stabilize the axio-dilaton is to take non-perturbative corrections to the superpotential into account. Whereas we have argued in section 2 that nonperturbative effects do not affect our supersymmetric solutions discussed before, one generically expects such corrections in models with more than one hypermultiplet. However, as pointed out above, if we turn on only $H_{R R}$ flux, we can avoid orientifolding and then there are more possibilities for non-perturbative corrections even in our models. (In type IIA language, we have twice as many three-cycles to wrap membranes if we do not orientifold.) The superpotential could then take the schematic form

$$
W=W_{R R}+A e^{i a \tau}
$$

where $A$ and $a$ are in principle functions of the vector multiplet moduli. As a result of pushing down the previous vacua with non-perturbative effects, AdS type vacua with a stabilized axio-dilaton emerge! It is interesting to uplift these solutions to dS, so that the masses of moduli fields become large enough. This can again be done with perturbative corrections to the Kähler potential.

\section{Conclusions and Open Questions}

One of the simplest models in which moduli stabilization can be studied emerges when the type IIB theory is compactified on an internal non-geometric theory for which Kähler moduli are absent. Such a model was constructed in terms of a LG model

\footnotetext{
${ }^{9}$ The factor 4 in the dilaton Kähler potential is important to achieve this.
} 
in [5]. Since the flux superpotential for the type IIB theory contains all the complex structure moduli and the dilaton, one could anticipate that the moduli can be stabilized at the classical level, i.e. in terms of flux only. The resulting theory is then extremely well under control because the anonymousness connected with non-perturbative effects coming from branes is lacking. In [5] it was shown that Minkowski as well AdS solutions at strong coupling with all moduli stabilized by fluxes can be found. Even though these vacua live at strong coupling, their existence is guaranteed due to the nonrenormalization of the type IIB flux superpotential.

In order to calculate physically relevant quantities for these vacua, such as the mass matrix, it is important to find weak coupling solutions. In this paper we have shown that four-dimensional Minkowski solutions live at strong coupling, so that the only Minkowski solutions of our model are the ones presented in [5]. We have also shown that weak coupling solutions (large value of the complex structure and small value for the dilaton) of AdS type exist. For a particular choice of flux configuration our model is mirror to the type IIA model of [4].

We have computed the mass matrix of the AdS type weak coupling solutions and we have seen that the masses of the states are proportional to the four-dimensional cosmological constant. Being of the order of the cosmological constant, one might be worried that this implies that the masses of moduli are too small. However we anticipated that this situation would change once supersymmetry is broken and the model is uplifted to four-dimensional $\mathrm{dS}$ space. We have discussed that perturbative corrections to the Kähler potential coming from higher order interactions in the type IIB theory break supersymmetry spontaneously. Once the vacua are uplifted to dS space the moduli become heavy.

We further illustrated the existence of a simple supersymmetry breaking solution for which $H_{N S}=0$, so that no constraints are imposed by the tadpole. Non-perturbative corrections to the superpotential (if they exist) could stabilize the dilaton, while perturbative corrections to the Kähler potential could uplift these vacua to dS space.

There are several rather interesting open questions. We have considered the technically simpler case of a $T^{6}$ with three equal complex structures. It would be interesting to construct the generic weak coupling solutions, i.e. the solution for three arbitrary complex structures and to check if the masses of moduli are of order of the cosmological constant. We have conjectured that this is generically the case in all models where parametric control can be achieved, so that this would constitute a check of our 
conjecture.

Since our Minkowski vacua (as well as many other flux vacua in the literature) live at strong coupling, an important open question is to understand if there exists a weakly coupled dual gauge theory describing these vacua. Some earlier attempts to find a dual gauge theory for the vacua of [4] were made in [35], where the precise form of this gauge theory was nevertheless not found. In this regard, it might be interesting to explore if a connection to the recently constructed four-dimensional Chern-Simons gauge theory in AdS can be found [36], 37.

As opposed to what happens in KKLT, where free parameters coming from the number of branes and anti-branes are present, in our model there are no free constants, as these are determined by the value of the corrections to the Kähler potential and the superpotential. It will be fascinating, though probably a hard job, to compute the exact numerical value of these constants. This teaches us that in string theory there are no free lunches!

Acknowledgments We would especially like to thank Shamit Kachru and Cumrun Vafa for many interesting discussions and comments on our manuscript. We further would like to thank Tom Banks, Aaron Bergmann, Yu-Chieh Chung, Michael Dine, Guangyu Guo, Simeon Hellerman, Joe Polchinski, Giovanni Villadoro, Stefan Vandoren, Edward Witten and Xi Yin for valuable discussions and communications. The work of K.B. was supported in part by NSF grants PHY-0505757 and the University of Texas A\&M. The work of M.B. was supported by NSF grants PHY-0505757 and the University of Texas A\&M. The work of J.W. was supported in part by the Roger Dashen Membership at the Institute for Advanced Study and by the NSF under grant number PHY-0503584. K.B. would like to thank the Institute for Advanced Study at Princeton, the Galileo Galilei Institute for Theoretical Physics and the CERN theory division for hospitality and financial support at different stages of this work. M.B. would like to thank the Harvard department of physics and the Institute for Advanced Study at Princeton for hospitality and financial support while this work was carried out. 


\section{References}

[1] M. Grana, "Flux compactifications in string theory: A comprehensive review," Phys. Rept. 423, 91 (2006), arXiv:hep-th/0509003.

[2] M. R. Douglas and S. Kachru, "Flux compactification," arXiv:hep-th/0610102.

[3] M. Dine and N. Seiberg, "Is The Superstring Weakly Coupled?," Phys. Lett. B 162, 299 (1985).

[4] O. DeWolfe, A. Giryavets, S. Kachru and W. Taylor, "Type IIA moduli stabilization," JHEP 0507, 066 (2005), arXiv:hep-th/0505160].

[5] K. Becker, M. Becker, C. Vafa and J. Walcher, "Moduli stabilization in non-geometric backgrounds," Nucl. Phys. B 770, 1 (2007) [arXiv:hep-th/0611001].

[6] C. Vafa, "The string landscape and the swampland," arXiv:hep-th/0509212.

[7] H. Ooguri and C. Vafa, "On the geometry of the string landscape and the swampland," Nucl. Phys. B 766, 21 (2007), arXiv:hep-th/0605264.

[8] S. Gukov, H. Ooguri and C. Vafa, unpublished.

[9] B. S. Acharya and M. R. Douglas, "A finite landscape?," arXiv:hep-th/0606212. M. R. Douglas, "Understanding the landscape," arXiv:hep-th/0602266.

[10] S. Kachru, R. Kallosh, A. Linde and S. P. Trivedi, "de Sitter vacua in string theory," Phys. Rev. D 68, 046005 (2003), arXiv:hep-th/0301240].

[11] S. Kachru, J. Pearson and H. L. Verlinde, "Brane/flux annihilation and the string dual of a non-supersymmetric field theory," JHEP 0206, 021 (2002) arXiv:hep-th/0112197.

[12] K. Becker, Y. C. Chung and G. Guo, to appear.

[13] M. Aganagic, C. Beem, J. Seo and C. Vafa, "Geometrically induced metastability and holography,", arXiv:hep-th/0610249.

[14] J. J. Heckman, J. Seo and C. Vafa, "Phase structure of a brane/anti-brane system at large N," arXiv:hep-th/0702077.

[15] R. Kallosh and M. Soroush, "Issues in type IIA uplifting," arXiv:hep-th/0612057.

[16] F. Saueressig, U. Theis and S. Vandoren, "On de Sitter vacua in type IIA orientifold compactifications," Phys. Lett. B 633, 125 (2006) arXiv:hep-th/0506181.

[17] K. Becker, M. Becker, M. Haack and J. Louis, "Supersymmetry breaking and alpha'corrections to flux induced potentials," JHEP 0206, 060 (2002), arXiv:hep-th/0204254.

[18] M. B. Green and M. Gutperle, "Effects of D-instantons," Nucl. Phys. B 498, 195 (1997), arXiv:hep-th/9701093.

[19] N. Berkovits and C. Vafa, "Type IIB R**4 $\mathrm{H}^{* *}(4 \mathrm{~g}-4)$ conjectures," Nucl. Phys. B 533, 181 (1998), arXiv:hep-th/9803145.

[20] K. Becker, M. Becker and A. Strominger, "Five-Branes, Membranes And Nonperturbative String Theory,” Nucl. Phys. B 456, 130 (1995), arXiv:hep-th/9507158. 
[21] M. Marino, R. Minasian, G. W. Moore and A. Strominger, "Nonlinear instantons from supersymmetric p-branes," JHEP 0001, 005 (2000), arXiv:hep-th/9911206.

[22] C. Vafa, "Superstrings and topological strings at large N," J. Math. Phys. 42, 2798 (2001) arXiv:hep-th/0008142.

[23] R. Dijkgraaf and C. Vafa, "A perturbative window into non-perturbative physics," arXiv:hep-th/0208048].

[24] C. P. Burgess, C. Escoda and F. Quevedo, "Nonrenormalization of flux superpotentials in string theory," JHEP 0606, 044 (2006), arXiv:hep-th/0510213.

[25] C. Vafa, "String Vacua and Orbifoldized L-G Models," Mod. Phys. Lett. A 4, 1169 (1989).

[26] I. Antoniadis, H. Partouche and T. R. Taylor, "Spontaneous Breaking of N=2 Global Supersymmetry," Phys. Lett. B 372, 83 (1996), arXiv:hep-th/9512006.

[27] T. R. Taylor and C. Vafa, "RR flux on Calabi-Yau and partial supersymmetry breaking," Phys. Lett. B 474, 130 (2000), arXiv:hep-th/9912152.

[28] E. A. Ivanov and B. M. Zupnik, "Modified $\mathrm{N}=2$ supersymmetry and FayetIliopoulos terms," Phys. Atom. Nucl. 62, 1043 (1999) [Yad. Fiz. 62, 1110 (1999)], arXiv:hep-th/9710236.

[29] P. Candelas, E. Derrick and L. Parkes, "Generalized Calabi-Yau manifolds and the mirror of a rigid manifold," Nucl. Phys. B 407, 115 (1993), arXiv:hep-th/9304045.

[30] P. Candelas, X. C. De La Ossa, P. S. Green and L. Parkes, "A pair of Calabi-Yau manifolds as an exactly soluble superconformal theory," Nucl. Phys. B 359, 21 (1991).

[31] W. Lerche, D. J. Smit and N. P. Warner, "Differential equations for periods and flat coordinates in two-dimensional topological matter theories," Nucl. Phys. B 372, 87 (1992), arXiv:hep-th/9108013.

[32] S. B. Giddings, S. Kachru and J. Polchinski, "Hierarchies from fluxes in string compactifications," Phys. Rev. D 66, 106006 (2002), [arXiv:hep-th/0105097].

[33] T. W. Grimm and J. Louis, "The effective action of type IIA Calabi-Yau orientifolds," Nucl. Phys. B 718, 153 (2005) arXiv:hep-th/0412277.

[34] T. W. Grimm, "The effective action of type II Calabi-Yau orientifolds," Fortsch. Phys. 53, 1179 (2005) arXiv:hep-th/0507153.

[35] T. Banks and K. van den Broek, "Massive IIA flux compactifications and U-dualities," JHEP 0703, 068 (2007), arXiv:hep-th/0611185].

[36] J. H. Schwarz, "Superconformal Chern-Simons theories," JHEP 0411, 078 (2004), arXiv:hep-th/0411077.

[37] D. Gaiotto and X. Yin, "Notes on superconformal Chern-Simons-matter theories," arXiv:hep-th/0704.3740. 